November 9, 2018

\title{
SCOTT'S PROBLEM FOR PROPER SCOTT SETS
}

\author{
VICTORIA GITMAN
}

\begin{abstract}
Some 40 years ago, Dana Scott proved that every countable Scott set is the standard system of a model of PA. Two decades later, Knight and Nadel extended his result to Scott sets of size $\omega_{1}$. Here, I show that assuming the Proper Forcing Axiom (PFA), every proper Scott set is the standard system of a model of PA. I define that a Scott set $\mathfrak{X}$ is proper if it is arithmetically closed and the quotient Boolean algebra $\mathfrak{X} / F$ in is a proper partial order. I also investigate the question of the existence of proper Scott sets.
\end{abstract}

\section{INTRODUCTION}

In this paper, I use the Proper Forcing Axiom (PFA) to make partial progress on a half-century-old question in the folklore of models of Peano Arithmetic about whether every Scott set is the standard system of a model of PA. The Proper Forcing Axiom is a generalization of Martin's Axiom that has found application in many areas of set theory in recent years. I will show that, assuming PFA, every arithmetically closed Scott set whose quotient Boolean algebra $\mathfrak{X} /$ Fin is proper is the standard system of a model of PA.

I will begin with some technical and historical details. We can associate to every model $M$ of PA a certain collection of subsets of the natural numbers called its standard system, in short $\operatorname{SSy}(M)$. The natural numbers $\mathbb{N}$ form the initial segment of every model of PA. The standard system consists of sets that arise as intersections of the definable (with parameters) sets of the model with the standard part $\mathbb{N}$. One thinks of the standard system as the traces left by the definable sets of the model on the natural numbers. A different way of characterizing sets in the standard system uses the notion of coding. Let us say that a set $A \subseteq \mathbb{N}$ is coded in a model $M$ if $M$ has an element $a$ such that $(a)_{n}=1$ if and only if $n \in A$. Here, $(a)_{x}$ can refer to any of the reader's favorite methods of coding with elements of a model of PA, e.g., by defining $(a)_{x}$ as the $x^{\text {th }}$ digit in the binary expansion of $a$. It is easy to see that for nonstandard models we can equivalently define the standard system to be the collection of all subsets of the natural numbers coded in the model.

What features characterize standard systems? Without reference to models of arithmetic, a standard system is just a particular collection of subsets of the natural numbers. Can we come up with a list of elementary (set theoretic, computability theoretic, etc.) properties that $\mathfrak{X} \subseteq \mathcal{P}(\mathbb{N})$ must satisfy in order to be the standard system of some model of PA? The notion of a Scott set encapsulates three key features of standard systems.

Definition 1.1. $\mathfrak{X} \subseteq \mathcal{P}(\mathbb{N})$ is a Scott set if

(1) $\mathfrak{X}$ is a Boolean algebra of sets.

I would like to thank my Ph.D. advisor Joel David Hamkins, Roman Kossak, and Ali Enayat for their many helpful suggestions. 
(2) If $A \in \mathfrak{X}$ and $B$ is Turing computable from $A$, then $B \in \mathfrak{X}$. 1

(3) If $T$ is an infinite binary tree coded by a set in $\mathfrak{X}$, then $\mathfrak{X}$ has a set coding some path through $T$.

It is relatively easy to see that every standard system is a Scott set (see [?], p. 175). Conversely, Dana Scott proved in 1962 that every countable Scott set is the standard system of a model of PA [?]. The proof relies on the fact that Scott sets are powerful enough to carry out internally the Henkin construction used to prove the Completeness Theorem. A crucial fact used in the proof is that if a set in the Scott set codes a consistent theory (again using the reader's favorite coding), then the Scott set must contain some completion of that theory as well. This follows easily if one is familiar with the relationship between building completions of a theory and branches through binary trees (see [?] p. 177-182 for a modern version of Scott's proof). So as the first step toward characterizing standard systems, we have:

Theorem 1.2 (Scott, 1962). Every countable Scott set is the standard system of a model of $\mathrm{PA}$.

Thus, countable Scott sets are exactly the countable standard systems of models of PA. Scott's theorem leads naturally to the following folklore question:

Scott's Problem. Is every Scott set the standard system of a model of PA?

In 1982, Knight and Nadel settled the question for Scott sets of size $\omega_{1}$.

Theorem 1.3 (Knight and Nadel, 1982). Every Scott set of size $\omega_{1}$ is the standard system of a model of PA. [?]

It follows that Scott sets of size $\omega_{1}$ are exactly the standard systems of size $\omega_{1}$ of models of PA. I will give a proof of Theorem 1.3 shortly.

Corollary 1.4. If $\mathrm{CH}$ holds, then Scott's Problem has a positive answer.

Very little is known about Scott's Problem if $\mathrm{CH}$ fails, that is, for Scott sets of size larger than $\omega_{1}$. I will use the techniques of forcing together with forcing axioms to find new conditions under which given a Scott set we can build a model of PA with that Scott set as the standard system. Given a Scott set $\mathfrak{X}$, we obtain a partial order $\mathfrak{X} /$ Fin consisting of the infinite sets in $\mathfrak{X}$ under the ordering of almost inclusion. Let us call a Scott set $\mathfrak{X}$ proper if it is arithmetically closed and the poset $\mathfrak{X} /$ Fin is proper (see Section 2 for the definition of properness and PFA and Section 3 for the significance of arithmetic closure). My main theorem is:

Main Theorem. Assuming PFA, every proper Scott set is the standard system of a model of $\mathrm{PA}$.

I will prove the main theorem by first generalizing a theorem known as Ehrenfeucht's Lemma to uncountable models using PFA. Ehrenfeucht's Lemma is an unpublished result of Ehrenfeucht from around the 1970's 2. One can use Ehrenfeucht's Lemma to give a proof of Theorem 1.3. Similarly, my generalized version is used to prove the main theorem. It is instructive to see a proof of Ehrenfeucht's

\footnotetext{
${ }^{1}$ Conditions (1) and (2) together imply that if $A_{1}, \ldots A_{n} \in \mathfrak{X}$ and $B$ is computable from $A_{1} \oplus \cdots \oplus A_{n}$, then $B \in \mathfrak{X}$.

${ }^{2}$ Roman Kossak, personal communication.
} 
Lemma to understand the difficulties involved in extending it to uncountable models (the proof below follows [?]).

Theorem 1.5 (Ehrenfeucht's Lemma). If $M$ is a countable model of PA whose standard system is contained in a Scott set $\mathfrak{X}$, then for any $A \in \mathfrak{X}$ there is an elementary extension $M \prec N$ such that $A \in \operatorname{SSy}(N) \subseteq \mathfrak{X}$.

Proof. First, we consider nonstandard $M$. Let $\mathfrak{X}$ be a Scott set such that $\operatorname{SSy}(M) \subseteq$ $\mathfrak{X}$ and let $A \in \mathfrak{X}$. Choose a countable Scott set $\mathfrak{Y} \subseteq \mathfrak{X}$ containing $\operatorname{SSy}(M)$ and $A$. Using the truth predicate for $\Sigma_{n}$-formulas, we can prove that the $\Sigma_{n}$-theory of $M$ is coded by a set in $\operatorname{SSy}(M)$ for every $n$. Moreover, all computable theories are in $\operatorname{SSy}(M)$ since $\operatorname{SSy}(M)$ is a Scott set. Therefore, the theory $T:=$ "PA $+\Sigma_{1}$-theory of $M$ " is in $\operatorname{SSy}(M)$. The idea that the Henkin construction can be carried out inside a Scott gives more than just Theorem 1.2, From this it follows that for any theory $T \supseteq$ PA such that $T \cap \Sigma_{n} \in \mathfrak{X}$ for every $n \in \mathbb{N}$, there exists a model of $T$ with that Scott set as the standard system. In particular, we get a model $M^{*}$ of $T$ with $\operatorname{SSy}\left(M^{*}\right)=\mathfrak{Y}$. By Friedman's Embedding Theorem (see [?], p. 161), since $M^{*} \models$ " $\Sigma_{1}$-theory of $M$ " and $\operatorname{SSy}(M) \subseteq \mathfrak{Y}$, we have $M \prec_{\Delta_{0}} M^{*}$. Close $M$ under initial segment and call the resulting submodel $N$. Then $M \prec N$ since it is cofinal and $\Delta_{0}$-elementary (by Gaifman's Embedding Theorem, see [?], p. 87). But also $\operatorname{SSy}(N)=\operatorname{SSy}\left(M^{*}\right)=\mathfrak{Y}$ as required since $N$ is an initial segment of $M^{*}$. This completes the proof for nonstandard models. Let TA $:=\{\varphi \mid \mathbb{N} \models \varphi\}$ denote True Arithmetic. It is clear that $\mathbb{N} \prec N$ if and only if $N \models$ TA. The standard system of $\mathbb{N}$ is the collection of all arithmetic sets. So suppose that $\mathfrak{X}$ is a Scott set containing all arithmetic sets and fix $A \in \mathfrak{X}$. It follows that TA $\cap \Sigma_{n}$ is in $\mathfrak{X}$ for every $n \in \mathbb{N}$. Let $\mathfrak{Y} \subseteq \mathfrak{X}$ be a countable Scott set containing $A$ and TA $\cap \Sigma_{n}$ for every $n \in \mathbb{N}$. By the remark above, there exists a model $N \models$ TA whose standard system is exactly $\mathfrak{Y}$. Thus, $\mathbb{N} \prec N$ and $A \in \operatorname{SSy}(N) \subseteq \mathfrak{X}$.

We are now ready to prove Knight and Nadel's result. 3

Proof of Theorem 1.3. Let $\mathfrak{X}$ be a Scott set of size $\omega_{1}$ and enumerate $\mathfrak{X}=\left\{A_{\xi} \mid\right.$ $\left.\xi<\omega_{1}\right\}$. The idea is to build up a model with the Scott set $\mathfrak{X}$ as the standard system in $\omega_{1}$ steps by successively throwing in one more set at each step and using Ehrenfeucht's Lemma to stay within $\mathfrak{X}$. More precisely, we will define an elementary chain $M_{0} \prec M_{1} \prec \cdots \prec M_{\xi} \prec \cdots$ of length $\omega_{1}$ of countable models of PA such that $\operatorname{SSy}\left(M_{\xi}\right) \subseteq \mathfrak{X}$ and $A_{\xi} \in \operatorname{SSy}\left(M_{\xi+1}\right)$. Then clearly $M=\cup_{\xi<\omega_{1}} M_{\xi}$ will work. Let $M_{0}$ be any countable model of PA with $\operatorname{SSy}\left(M_{0}\right) \subseteq \mathfrak{X}$. Such $M_{0}$ exists by Scott's theorem (1.2). Given $M_{\xi}$, by Ehrenfeucht's Lemma, there exists $M_{\xi+1}$ such that $M_{\xi} \prec M_{\xi+1}$, the set $A_{\xi} \in \operatorname{SSy}\left(M_{\xi+1}\right)$, and $\operatorname{SSy}\left(M_{\xi+1}\right) \subseteq \mathfrak{X}$. At limit stages take unions.

The key ideas in the proof of Theorem 1.3 can be summarized in the following definition and theorem:

Definition 1.6 (The $\kappa$-Ehrenfeucht Principle for $\Gamma$ ). Let $\kappa$ be a cardinal and $\Gamma$ some collection of Scott sets. The $\kappa$-Ehrenfeucht Principle for $\Gamma$ states that if $M$ is a model of PA of size less than $\kappa$ and $\mathfrak{X}$ is a Scott set in $\Gamma$ such that $\operatorname{SSy}(M) \subseteq \mathfrak{X}$, then for any $A \in \mathfrak{X}$ there is an elementary extension $M \prec N$ such that $A \in \operatorname{SSy}(N) \subseteq \mathfrak{X}$.

\footnotetext{
${ }^{3}$ This is not Knight and Nadel's original proof.
} 
If $\Gamma$ is the collection of all Scott sets, we will say simply that the $\kappa$-Ehrenfeucht Principle holds.

In view of Definition [1.6 Ehrenfeucht's Lemma (Theorem [1.5) is the $\omega_{1}$-Ehrenfeucht Principle. We can freely assume that the elementary extension $N$ given by the $\kappa$-Ehrenfeucht Principle has size less than $\kappa$ since if this is not the case, we can always take an elementary submodel $N^{\prime}$ of $N$ such that $M \prec N^{\prime}$ and $A \in \operatorname{SSy}\left(N^{\prime}\right)$. A completely straightforward generalization of the proof of Theorem 1.3 gives:

Theorem 1.7. If the $\kappa$-Ehrenfeucht Principle for $\Gamma$ holds, then every Scott set in $\Gamma$ of size $\kappa$ is the standard system of a model of PA.

Thus, one approach to solving Scott's Problem would be to try to prove the $\omega_{2}$-Ehrenfeucht Principle for some collection of Scott sets. However, proofs of Ehrenfeucht's Lemma hinge precisely on those techniques in the field of models of PA that appear to work only with countable models. As an example, Friedman's famous Embedding Theorem does not generalize to uncountable models. 4 In what follows, I will mainly investigate the Ehrenfeucht principles. The results on Scott's Problem will follow as a corollary. Under PFA, I will show that the $\omega_{2}$-Ehrenfeucht Principle for proper Scott sets holds (Theorem 3.4).

\section{Set Theory and Scott's Problem}

Since the result of Knight and Nadel (Theorem 1.3), very little progress had been made on Scott's Problem until some recent work of Fredrik Engström [?]. It is not difficult to believe that Scott's Problem past $\omega_{1}$ might have a set theoretic resolution. Engström followed a strategy, suggested more than a decade earlier by Joel Hamkins and others, to use forcing axioms to gain new insight into the problem. We saw that a positive answer to Scott's Problem follows from CH. It is a standard practice in set theoretic proofs that if a statement follows from $\mathrm{CH}$, we try to prove it or its negation from $\neg \mathrm{CH}+$ Martin's Axiom. Martin's Axiom (MA) is a forcing axiom which asserts that for every c.c.c. poset $\mathbb{P}$ and every collection $\mathcal{D}$ of less than the continuum many dense subsets of $\mathbb{P}$, there is a filter on $\mathbb{P}$ that meets all of them. Such filters are often called partially generic filters. Engström tried to use Martin's Axiom to find new techniques for building models of PA whose standard system is a given Scott set.

Given a Scott set $\mathfrak{X}$, Engström chose the poset $\mathfrak{X} /$ Fin, whose elements are infinite sets in $\mathfrak{X}$ ordered by almost inclusion. That is, for infinite $A$ and $B$ in $\mathfrak{X}$, we say that $A \leq B$ if and only if $A \subseteq_{\text {Fin }} B$. Observe that $\mathfrak{X} /$ Fin is forcing equivalent to forcing with the Boolean algebra $\mathfrak{X}$ modulo the ideal of finite sets. A familiar and thoroughly studied instance of this poset is $\mathcal{P}(\mathbb{N}) /$ Fin. A Scott set is arithmetically closed if whenever $A$ is in it and $B$ is arithmetically definable from $A$, then $B$ is also in it (for a more extensive discussion, see Section 3 ). For a property of posets $\mathscr{P}$, if $\mathfrak{X}$ is an arithmetically closed Scott set and $\mathfrak{X} /$ Fin has $\mathscr{P}$, I will simply say that $\mathfrak{X}$ has property $\mathscr{P}$. An important point to be noted here is that whenever a Scott set $\mathfrak{X}$ is discussed as a poset, I will always be assuming that it is arithmetically closed. The significance of arithmetic closure will become apparent in Section 3 .

\footnotetext{
${ }^{4}$ Here $\omega_{1}$-like models are an obvious counterexample.
} 
Theorem 2.1 (Engström, 2004). Assuming Martin's Axiom, every c.c.c. Scott set of size less than the continuum is the standard system of a model of PA. [?]

To obtain models for Scott sets for which we could not do so before, Engström needed that there are uncountable Scott sets that are c.c.c.. Unfortunately:

Theorem 2.2. Every c.c.c. Scott set is countable.

Proof. Let $\mathfrak{X}$ be a Scott set. If $x$ is a finite subset of $\mathbb{N}$, let $\ulcorner x\urcorner$ denote the code of $x$ using Gödel's coding. For every $A \in \mathfrak{X}$, define an associated $A^{\prime}=\{\ulcorner A \cap n\urcorner \mid n \in \mathbb{N}\}$. Clearly $A^{\prime}$ is computable from $A$, and hence in $\mathfrak{X}$. Observe that if $A \neq B$, then $\left|A^{\prime} \cap B^{\prime}\right|<\omega$. Hence if $A \neq B$, we get that $A^{\prime}$ and $B^{\prime}$ are incompatible in $\mathfrak{X} /$ Fin. It follows that $\mathscr{A}=\left\{A^{\prime} \mid A \in \mathfrak{X}\right\}$ is an antichain of $\mathfrak{X} /$ Fin of size $|\mathfrak{X}|$. This shows that $\mathfrak{X} /$ Fin always has antichains as large as the whole poset.

Thus, the poset $\mathfrak{X} /$ Fin has the worst possible chain condition, namely $|\mathfrak{X}|^{+}$-c.c.. Theorem 2.2 implies that no new instances of Scott's Problem can be obtained from Theorem 2.1.

I will borrow from Engström's work the poset $\mathfrak{X} /$ Fin. But my strategy will be different in two respects. First, instead of MA, I will use the poset together with the forcing axiom PFA, allowing me to get around the obstacle of Theorem 2.2 In Section 7, I will argue that, unlike the case with c.c.c. Scott sets, uncountable proper Scott sets do exist. However, I will not be able to explicitly obtain any new instances of Scott's Problem. Second, my main aim will be to obtain an extension of Ehrenfeucht's Lemma to uncountable models, while Engström's was to directly get a model whose standard system is a given Scott set. This approach will allow me to handle Scott sets of size continuum, which had not been possible with Engström's techniques.

Recall that for a cardinal $\lambda$, the set $H_{\lambda}$ is the collection of all sets whose transitive closure has size less than $\lambda$. Let $\mathbb{P}$ be a poset and $\lambda$ be a cardinal greater than $2^{|\mathbb{P}|}$. Since we can always take an isomorphic copy of $\mathbb{P}$ on the cardinal $|\mathbb{P}|$, we can assume without loss of generality that $\mathbb{P}$ and $\mathcal{P}(\mathbb{P})$ are elements of $H_{\lambda}$. In particular, we want to ensure that all dense subsets of $\mathbb{P}$ are in $H_{\lambda}$. Let $M$ be a countable elementary submodel of $H_{\lambda}$ containing $\mathbb{P}$ as an element. If $G$ is a filter on $\mathbb{P}$, we say that $G$ is $M$-generic if for every maximal antichain $A \in M$ of $\mathbb{P}$, the intersection $G \cap A \cap M \neq \emptyset$. It must be explicitly specified what $M$-generic means in this context since the usual notion of generic filters makes sense only for transitive structures and $M$ is not necessarily transitive. This definition of $M$-generic is closely related to the definition for transitive structures. To see this, let $M^{*}$ be the Mostowski collapse of $M$ and $\mathbb{P}^{*}$ be the image of $\mathbb{P}$ under the collapse. Let $G^{*} \subseteq \mathbb{P}^{*}$ be the pointwise image of $G \cap M$ under the collapse. Then $G$ is $M$-generic if and only if $G^{*}$ is $M^{*}$-generic for $\mathbb{P}^{*}$ in the usual sense.

Definition 2.3. Let $\mathbb{P} \in H_{\lambda}$ be a poset and $M$ be an elementary submodel of $H_{\lambda}$ containing $\mathbb{P}$. Then a condition $q \in \mathbb{P}$ is $M$-generic if and only if every $V$-generic filter $G \subseteq \mathbb{P}$ containing $q$ is $M$-generic.

Definition 2.4. A poset $\mathbb{P}$ is proper if for every $\lambda>2^{|\mathbb{P}|}$ and every countable $M \prec H_{\lambda}$ containing $\mathbb{P}$, for every $p \in \mathbb{P} \cap M$, there is an $M$-generic condition below $p$. 
It can be shown that it is actually equivalent to consider only some fixed $\lambda>2^{|\mathbb{P}|}$ and to show that generic conditions exist only for a club of countable $M \prec H_{\lambda}[?]$ (p. 102).

Definition 2.5. The Proper Forcing Axiom (PFA) is the assertion that for every proper poset $\mathbb{P}$ and every collection $\mathcal{D}$ of at most $\omega_{1}$ many dense subsets of $\mathbb{P}$, there is a filter on $\mathbb{P}$ that meets all of them.

Proper forcing was invented by Shelah, who sought a class of $\omega_{1}$-preserving forcing notions that would be preserved under countable support iterations (for an introduction to proper forcing see [?] (p. 601) or [?]). The two familiar classes of $\omega_{1}$-preserving forcing notions, namely the c.c.c. and countably closed forcing notions, turn out to be proper as well. The Proper Forcing Axiom, introduced by Baumgartner [?], is easily seen to be a generalization of Martin's Axiom since c.c.c. posets are proper and PFA decides the size of the continuum is $\omega_{2}$. The later fact is a highly nontrivial result in [?]. In many respects, however, PFA is very much unlike MA. Not only does it decide the size of the continuum, the axiom also has large cardinal strength. The best known large cardinal upper bound on the consistency of PFA is a supercompact cardinal [?]. Much fruitful set theoretical work in recent years has involved PFA and its consequences.

\section{Proof of the Main Theorem}

I will use PFA to prove the $\omega_{2}$-Ehrenfeucht Principle for proper Scott sets. The main theorem will follow as a corollary.

A filter $G$ on the poset $\mathfrak{X} /$ Fin is easily seen to be a filter on the Boolean algebra $\mathfrak{X}$. By extending $G$ to a larger filter if necessary, we can assume without loss of generality that $G$ is an ultrafilter. Recall that to prove the $\omega_{2}$-Ehrenfeucht Principle, given a model $M$ of size $\omega_{1}$ and a Scott set $\mathfrak{X}$ such $\mathrm{SSy}(\mathrm{M}) \subseteq \mathfrak{X}$, we need to find for every $A \in \mathfrak{X}$, an elementary extension $N$ such that $A \in \operatorname{SSy}(N) \subseteq \mathfrak{X}$. The strategy will be to find $\omega_{1}$ many dense subsets of $\mathfrak{X} /$ Fin such that if $G$ is a partially generic ultrafilter meeting all of them, then the standard system of the ultrapower of $M$ by $G$ will stay within $\mathfrak{X}$. Thus, if $\mathfrak{X}$ is proper, we will be able to use PFA to obtain such an ultrafilter. I will also show that to every $A \in \mathfrak{X}$, there corresponds a set $B \in \mathfrak{X} /$ Fin such that whenever $B$ is in an ultrafilter $G$, the set $A$ will end up in the ultrapower of $M$ by $G$.

Let $\mathcal{S} \subseteq \mathcal{P}(\mathbb{N})$ and expand the language of arithmetic $\mathcal{L}_{A}$ to include unary predicates for all $A \in \mathcal{S}$. Then the structure $\mathbb{N}_{\mathcal{S}}=\langle\mathbb{N}, A\rangle_{A \in \mathcal{S}}$ is a structure of this expanded language with the natural interpretation. Since Scott sets are closed under relative computability, basic computability theory arguments show that if $\mathfrak{X}$ is a Scott set, the structure $\mathbb{N}_{\mathfrak{X}}=\langle\mathbb{N}, A\rangle_{A \in \mathfrak{X}}$ is closed under $\Delta_{1}$-definability. That is, if $B$ is $\Delta_{1}$-definable in $\mathbb{N}_{\mathfrak{X}}$, then $B \in \mathfrak{X}$.

Definition 3.1. A collection $\mathcal{S} \subseteq \mathcal{P}(\mathbb{N})$ is arithmetically closed if the structure $\mathbb{N}_{\mathcal{S}}=\langle\mathbb{N}, A\rangle_{A \in \mathcal{S}}$ is closed under definability. That is, if $B$ is definable in $\mathbb{N}_{\mathcal{S}}$, then $B \in \mathcal{S}$.

A Scott set $\mathfrak{X}$ is arithmetically closed simply when it satisfies Definition 3.1. Observe actually that if $\mathcal{S}$ is arithmetically closed, then it is a Scott set. Thus, arithmetic closure subsumes the definition of a Scott set. An easy induction on the complexity of formulas establishes that if $\mathfrak{X}$ is a Boolean algebra of sets and 
$\mathbb{N}_{\mathfrak{X}}=\langle\mathbb{N}, A\rangle_{A \in \mathfrak{X}}$ is closed under $\Sigma_{1}$-definability, then $\mathfrak{X}$ is arithmetically closed. Hence a Scott set is arithmetically closed if and only if it is closed under the Turing jump operation.

Definition 3.2. Say that $\left\langle B_{n} \mid n \in \mathbb{N}\right\rangle$ is coded in $\mathfrak{X}$ if there is $B \in \mathfrak{X}$ such that $B_{n}=\{m \in \mathbb{N} \mid\langle n, m\rangle \in B\}$. Given $\left\langle B_{n} \mid n \in \mathbb{N}\right\rangle$ coded in $\mathfrak{X}$ and $C \in \mathfrak{X} /$ Fin, say that $C$ decides $\left\langle B_{n} \mid n \in \mathbb{N}\right\rangle$ if whenever $U$ is an ultrafilter on $\mathfrak{X}$ and $C \in U$, then $\left\{n \in \mathbb{N} \mid B_{n} \in U\right\} \in \mathfrak{X}$. Call a Scott set $\mathfrak{X}$ decisive if for every $\left\langle B_{n} \mid n \in \mathbb{N}\right\rangle$ coded in $\mathfrak{X}$, the set $\mathscr{D}=\{C \in \mathfrak{X} /$ Fin $\mid C$ decides $\left.\left\langle B_{n} \mid n \in \mathbb{N}\right\rangle\right\}$ is dense in $\mathfrak{X} /$ Fin.

Decisiveness is precisely the property of a Scott set which is required for our proof of the main theorem. I will show below that decisiveness is equivalent to arithmetic closure.

Lemma 3.3. The following are equivalent for a Scott set $\mathfrak{X}$ :

(1) $\mathfrak{X}$ is arithmetically closed.

(2) $\mathfrak{X}$ is decisive.

(3) For every sequence $\left\langle B_{n} \mid n \in \mathbb{N}\right\rangle$ coded in $\mathfrak{X}$, there is $C \in \mathfrak{X} /$ Fin deciding $\left\langle B_{n} \mid n \in \mathbb{N}\right\rangle$.

Proof.

$(1) \Longrightarrow(2)$. 5 Assume that $\mathfrak{X}$ is arithmetically closed. Fix $A \in \mathfrak{X} /$ Fin and a sequence $\left\langle B_{n} \mid n \in \mathbb{N}\right\rangle$ coded in $\mathfrak{X}$. We need to show that there is an element in $\mathfrak{X} /$ Fin below $A$ deciding $\left\langle B_{n} \mid n \in \mathbb{N}\right\rangle$. For every finite binary sequence $s$, we will define $B_{s}$ by induction on the length of $s$. Let $B_{\emptyset}=A$. Given $B_{s}$, where $s$ has length $n$, define $B_{s 1}=B_{s} \cap B_{n}$ and $B_{s 0}=B_{s} \cap\left(\mathbb{N}-B_{n}\right)$. Define the binary tree $T=\{s \in$ $2^{<\omega} \mid B_{s}$ is infinite $\}$. Clearly $T$ is infinite since if we split an infinite set into two pieces one of them must still be infinite. Since $\mathfrak{X}$ is arithmetically closed and $T$ is arithmetic in $A$ and $\left\langle B_{n} \mid n \in \mathbb{N}\right\rangle$, it follows that $T \in \mathfrak{X}$. Thus, $\mathfrak{X}$ contains a cofinal branch $P$ through $T$. Define $C=\left\{b_{n} \mid n \in \mathbb{N}\right\}$ such that $b_{0}$ is least element of $B_{\emptyset}$ and $b_{n+1}$ is least element of $B_{P \uparrow n}$ that is greater than $b_{n}$. Clearly $C$ is infinite and $C \subseteq A$. Now suppose $U$ is an ultrafilter on $\mathfrak{X}$ and $C \in U$, then $B_{n} \in U$ if and only if $C \subseteq_{\text {Fin }} B_{n}$. Thus, $\left\{n \in \mathbb{N} \mid B_{n} \in U\right\}=\left\{n \in \mathbb{N} \mid C \subseteq_{\text {Fin }} B_{n}\right\} \in \mathfrak{X}$ since $\mathfrak{X}$ is arithmetically closed.

$(2) \Longrightarrow(3)$ : Clear.

$(3) \Longrightarrow(1) \sqrt{6}$ : It suffices to show that $\mathfrak{X}$ is closed under the Turing jump operation. Fix $A \in \mathfrak{X}$ and define the sequence $\left\langle B_{n} \mid n \in \mathbb{N}\right\rangle$ by $k \in B_{n}$ if and only if the Turing program coded by $n$ with oracle $A$ halts on input $n$ in less than $k$ many steps. Clearly the sequence is computable from $A$, and hence coded in $\mathfrak{X}$. Let $H=\{n \in \mathbb{N} \mid$ the program coded by $n$ with oracle $A$ halts on input $n\}$ be the halting problem for $A$. It should be clear that $n \in H$ implies that $B_{n}$ is cofinite and $n \notin H$ implies that $B_{n}=\emptyset$. Let $C \in \mathfrak{X} /$ Fin deciding $\left\langle B_{n} \mid n \in \mathbb{N}\right\rangle$ and $U$ be any ultrafilter containing $C$, then $\left\{n \in \mathbb{N} \mid B_{n} \in U\right\} \in \mathfrak{X}$. But this set is exactly $H$. This shows that $H \in \mathfrak{X}$, and hence $\mathfrak{X}$ is closed under the Turing jump operation.

Theorem 3.4. Assuming PFA, the $\omega_{2}$-Ehrenfeucht Principle for proper Scott sets holds. That is, if $\mathfrak{X}$ is a proper Scott set and $M$ is a model of $\mathrm{PA}$ of size $\omega_{1}$ whose

\footnotetext{
${ }^{5}$ Similar arguments have appeared in [?] and other places.

${ }^{6}$ I am grateful to Joel Hamkins for pointing out this argument.
} 
standard system is contained in $\mathfrak{X}$, then for any $A \in \mathfrak{X}$, there is an elementary extension $M \prec N$ such that $A \in \operatorname{SSy}(N) \subseteq \mathfrak{X}$.

Proof. I will build $N$ using a variation on the ultrapower construction introduced by Kirby and Paris [?]. Fix a model $M$ of PA and a Scott set $\mathfrak{X}$ such that $\operatorname{SSy}(M) \subseteq \mathfrak{X}$. Let $G$ be some ultrafilter on $\mathfrak{X}$. If $f: \mathbb{N} \rightarrow M$, we say that $f$ is coded in $M$ when there is $a \in M$ such that $(a)_{n}=f(n)$ for all $n \in \mathbb{N}$. Given $f$ and $g$ coded in $M$, define $f \sim_{G} g$ if $\{n \in \mathbb{N} \mid f(n)=g(n)\} \in G$. The definition makes sense since clearly $\{n \in \mathbb{N} \mid f(n)=g(n)\} \in \operatorname{SSy}(M) \subseteq \mathfrak{X}$. The classical ultrapower construction uses an ultrafilter on $\mathcal{P}(\mathbb{N})$ and all functions from $\mathbb{N}$ to $M$. This construction uses only functions coded in $M$, and therefore needs only an ultrafilter on $\operatorname{SSy}(M) \subseteq \mathfrak{X}$. As in the classical construction, we get an equivalence relation and a well-defined $\mathcal{L}_{A}$ structure on the equivalence classes. The proof relies on the fact that $\mathfrak{X}$ is a Boolean algebra. Call $\Pi_{\mathfrak{X}} M / G$ the collection of equivalence classes $[f]_{G}$ where $f$ is coded in $M$. Also, as usual, we get:

Lemma 3.4.1. Eoś Lemma holds. That is, $\Pi_{\mathfrak{X}} M / G \models \varphi\left([f]_{G}\right)$ if and only if $\{n \in \mathbb{N} \mid M \models \varphi(f(n))\} \in G$.

Proof. Similar to the classical proof of the Łoś Lemma.

Lemma 3.4.2. For every $A \in \mathfrak{X}$, there is $B \in \mathfrak{X} /$ Fin such that if $G$ is any ultrafilter on $\mathfrak{X}$ containing $B$, then $A \in \operatorname{SSy}\left(\Pi_{\mathfrak{X}} M / G\right)$.

Proof. Let $\chi_{A}$ be the characteristic function of $A$. For every $n \in \mathbb{N}$, define $B_{n}=$ $\left\{m \in \mathbb{N} \mid(m)_{n}=\chi_{A}(n)\right\}$. Then clearly each $B_{n} \in \mathfrak{X}$ and $\left\langle B_{n} \mid n \in \mathbb{N}\right\rangle$ is coded in $\mathfrak{X}$ since the sequence is arithmetic in $A$. Observe that the intersection of any finite number of $B_{n}$ is infinite. Let $B=\left\{b_{n} \mid n \in \mathbb{N}\right\}$ where $b_{0}$ is least element of $B_{0}$ and $b_{n+1}$ is least element of $\cap_{m \leq n+1} B_{m}$ that is greater than $b_{n}$. Then $B \subseteq_{\text {Fin }} B_{n}$ for all $n \in \mathbb{N}$ and $B \in \mathfrak{X}$ since it is arithmetic in $\left\langle B_{n}: n \in \mathbb{N}\right\rangle$. It follows that if $G$ is any ultrafilter containing $B$, then $G$ must contain all the $B_{n}$ as well. Let $G$ be an ultrafilter containing $B$. Let $i d: \mathbb{N} \rightarrow \mathbb{N}$ be the identity function. I claim that $\left([i d]_{G}\right)_{n}=\chi_{A}(n)$. It will follow that $A \in \operatorname{SSy}\left(\Pi_{\mathfrak{X}} M / G\right)$. But this is true since $\left([i d]_{G}\right)_{n}=\chi_{A}(n)$ if and only if $\left\{m \in \mathbb{N} \mid(m)_{n}=\chi_{A}(n)\right\}=B_{n} \in G$.

Lemma 3.4 .2 tells us that if we want to add some set $A$ to the standard system of the ultrapower that we are building, we just have to make sure that a correct set gets put into the ultrafilter. It follows that that we can build ultrapowers of $M$ having any given element of $\mathfrak{X}$ in the standard system.

The crucial step of the construction is to find a family of size $\omega_{1}$ of dense subsets of $\mathfrak{X} /$ Fin such that if the ultrafilter meets all members of the family, the standard system of the ultrapower stays within $\mathfrak{X}$. It is in this step that we need the decisiveness of $\mathfrak{X}$.

Recall that a set $E$ is in the standard system of a nonstandard model if and only if there is an element $e$ such that $E=\left\{n \in \mathbb{N} \mid(e)_{n}=1\right\}$, meaning $E$ is coded in the model. Thus, we have to show that the sets coded by elements of $\Pi_{\mathfrak{X}} M / G$ are in $\mathfrak{X}$.

Lemma 3.4.3. For every function $f: \mathbb{N} \rightarrow M$ coded in $M$, there is a dense subset $\mathscr{D}_{f}$ of $\mathfrak{X} /$ Fin such that if $G$ meets $\mathscr{D}_{f}$, then $[f]_{G} \in \Pi_{\mathfrak{X}} M / G$ codes a set in $\mathfrak{X}$.

Proof. Fix a function $f: \mathbb{N} \rightarrow M$ coded in $M$ and let $E_{f}=\left\{n \in \mathbb{N} \mid \Pi_{\mathfrak{X}} M / G \models\right.$ $\left.\left([f]_{G}\right)_{n}=1\right\}$. By Loś Lemma, $\Pi_{\mathfrak{X}} M / G \models\left([f]_{G}\right)_{n}=1$ if and only if 
$\left\{m \in \mathbb{N} \mid(f(m))_{n}=1\right\} \in G$. Define $B_{n, f}=\left\{m \in \mathbb{N} \mid(f(m))_{n}=1\right\}$ and note that $\left\langle B_{n, f} \mid n \in \mathbb{N}\right\rangle$ is coded in $\operatorname{SSy}(M)$. Observe that $n \in E_{f}$ if and only if $B_{n, f} \in G$. Thus, we have to make sure that $\left\{n \in \mathbb{N} \mid B_{n, f} \in G\right\} \in \mathfrak{X}$. Let $\mathscr{D}_{f}=\left\{C \in \mathfrak{X} /\right.$ Fin $\mid C$ decides $\left.\left\langle B_{n, f} \mid n \in \mathbb{N}\right\rangle\right\}$. Since $\mathfrak{X}$ is decisive, $\mathscr{D}_{f}$ is dense. Clearly if $G$ meets $\mathscr{D}_{f}$, the set coded by $[f]_{G}$ will be in $\mathfrak{X}$.

Now we can finish the proof of Theorem 3.4. Let $\mathcal{D}=\left\{\mathscr{D}_{f} \mid f: \mathbb{N} \rightarrow M\right.$ is coded in $M\}$. Since $M$ has size $\omega_{1}$, the collection $\mathcal{D}$ has size $\omega_{1}$ also. Assuming PFA guarantees that we can find an ultrafilter $G$ meeting every $\mathscr{D}_{f} \in \mathcal{D}$. But this is precisely what forces the standard system of $\Pi_{\mathfrak{X}} M / G$ to stay inside $\mathfrak{X}$.

The main theorem now follows directly from Theorem 3.4

Proof of Main Theorem. Since PFA implies $2^{\omega}=\omega_{2}$ and Scott sets of size $\omega_{1}$ are already handled by Knight and Nadel's result, we only need to consider Scott sets of size $\omega_{2}$. But the result for these follows from Theorem 1.7 and the $\omega_{2}$-Ehrenfeucht Principle established by Theorem 3.4 .

\section{Extensions of Ehrenfeucht's Lemma}

Below, I will go through some results related to the question of extending Ehrenfeucht's Lemma to models of size $\omega_{1}$ ( $\omega_{2}$-Ehrenfeucht Principle).

Theorem 3.4 shows that in a universe satisfying PFA, the $\omega_{2}$-Ehrenfeucht Principle for proper Scott sets holds. Next, I will use the same techniques to show that the $\kappa$-Ehrenfeucht Principle for arithmetically closed Scott sets holds for all $\kappa$ if we only consider models with countable standard systems. For this argument, we do not need to use PFA or properness.

Theorem 4.1. If $M$ is a model of $\mathrm{PA}$ whose standard system is countable and contained in an arithmetically closed Scott set $\mathfrak{X}$, then for any $A \in \mathfrak{X}$, there is an elementary extension $M \prec N$ such that $A \in \operatorname{SSy}(N) \subseteq \mathfrak{X}$.

Proof. Fix an arithmetically closed Scott set $\mathfrak{X}$ and a model $M$ of PA such that $\operatorname{SSy}(M)$ is countable and contained in $\mathfrak{X}$. To mimic the proof of Theorem 3.4 we need to find an ultrafilter $G$ on $\mathfrak{X}$ which meets the dense sets $\mathscr{D}_{f}=\{C \in \mathfrak{X} /$ Fin $\mid$ $C$ decides $\left.\left\langle B_{n, f} \mid n \in \mathbb{N}\right\rangle\right\}$. I claim that there are only countably many $\mathscr{D}_{f}$. If this is the case, then such an ultrafilter exists without any forcing axiom assumption. Given $f: \mathbb{N} \rightarrow M$, let $B_{f}$ code $\left\langle B_{n, f} \mid n \in \mathbb{N}\right\rangle$. There are possibly as many $f$ as elements of $M$, but there can be only countably many $B_{f}$ since each $B_{f} \in \operatorname{SSy}(M)$. It remains only to observe that $\mathscr{D}_{f}$ is determined by $B_{f}$. So there are as many $\mathscr{D}_{f}$ as there are different $B_{f}$. Thus, there are only countably many $\mathscr{D}_{f}$ in spite of the fact that $M$ can be arbitrarily large.

The same idea can be used to extend Theorem 3.4 to show that the $\kappa$-Ehrenfeucht Principle for proper Scott sets holds for all $\kappa$ if we consider only models whose standard system has size $\omega_{1}$.

Theorem 4.2. Assuming PFA, if $\mathfrak{X}$ is a proper Scott set and $M$ is a model of $\mathrm{PA}$ whose standard system has size $\omega_{1}$ and is contained in $\mathfrak{X}$, then for any $A \in \mathfrak{X}$, there is an elementary extension $M \prec N$ such that $A \in \operatorname{SSy}(N) \subseteq \mathfrak{X}$.

It is also an easy consequence of an amalgamation result for models of PA that the $\kappa$-Ehrenfeucht Principle holds for all $\kappa$ for models with a countable nonstandard 
elementary initial segment. Neither PFA nor arithmetic closure is required for this result.

Theorem 4.3. Suppose $M_{0}, M_{1}$, and $M_{2}$ are models of PA such that $M_{0} \prec_{c o f} M_{1}$ and $M_{0} \prec$ end $M_{2}$. Then there is an amalgamation $M_{3}$ of $M_{1}$ and $M_{2}$ over $M_{0}$ such that $M_{1} \prec_{\text {end }} M_{3}$ and $M_{2} \prec$ cof $M_{3}$. (See [?], p. 40)

Theorem 4.4. Suppose $M$ is a model of $\mathrm{PA}$ with a countable nonstandard elementary initial segment and $\mathfrak{X}$ is a Scott set such that $\operatorname{SSy}(M) \subseteq \mathfrak{X}$. Then for any $A \in \mathfrak{X}$, there is an elementary extension $M \prec N$ such that $A \in \operatorname{SSy}(N) \subseteq \mathfrak{X}$.

Proof. Fix a set $A \in \mathfrak{X}$. Let $K$ be a countable nonstandard elementary initial segment of $M$, then $\operatorname{SSy}(K)=\operatorname{SSy}(M)$. By Ehrenfeucht's Lemma (Theorem 1.5), there is an extension $K \prec_{\text {cof }} K^{\prime}$ such that $A \in \operatorname{SSy}\left(K^{\prime}\right) \subseteq \mathfrak{X}$. By Theorem 4.4. there is a model $N$, an amalgamation of $K^{\prime}$ and $M$ over $K$, such that $K^{\prime} \prec_{\text {end }} N$ and $M \prec_{\text {cof }} N$. It follows that $\operatorname{SSy}\left(K^{\prime}\right)=\operatorname{SSy}(N)$. Thus, $A \in \operatorname{SSy}(N) \subseteq \mathfrak{X}$.

Corollary 4.5. The $\kappa$-Ehrenfeucht Principle holds for $\omega_{1}$-like models for all cardinals $\kappa$.

These observations suggest that if the $\omega_{2}$-Ehrenfeucht Principle fails to hold, one should look to models with an uncountable standard system for such a counterexample.

\section{Other Applichtions of $\mathfrak{X} /$ Fin}

It appears that $\mathfrak{X} /$ Fin is a natural poset to use in several unresolved questions in the field of models of PA. In the previous sections, I used it to find new conditions for extending Ehrenfeucht's Lemma and Scott's Problem. Here, I will mention some other instances in which the poset naturally arises.

Definition 5.1. Let $\mathcal{L}$ be some language extending the language of arithmetic $\mathcal{L}_{A}$. We say that a model $M$ of $\mathcal{L}$ satisfies $\mathrm{PA}^{*}$ if $M$ satisfies induction axioms in the expanded language. If $M \models \mathrm{PA}^{*}$, then $M \subseteq N$ is a conservative extension if it is a proper extension and every parametrically definable subset of $N$ when restricted to $M$ is also definable in $M$.

Gaifman showed in [?] that for any countable language $\mathcal{L}$, every $M \models \mathrm{PA}^{*}$ in $\mathcal{L}$ has a conservative elementary extension. A result of George Mills shows that the statement fails for uncountable languages. Mills proved that every countable nonstandard model $M \models \mathrm{PA}^{*}$ in a countable language has an expansion to an uncountable language such that $M \models \mathrm{PA}^{*}$ in the expanded language, but has no conservative elementary extension (see [?], p. 168). His techniques failed for the standard model, leaving open the question whether there is an expansion of the standard model $\mathbb{N}$ to some uncountable language that does not have a conservative elementary extension. This question has recently been answered by Ali Enayat, who demonstrated that there is always an uncountable arithmetically closed Scott set $\mathfrak{X}$ such that $\langle\mathbb{N}, A\rangle_{A \in \mathfrak{X}}$ has no conservative elementary extension [?]. This raises the question of whether we can say something general about Scott sets $\mathfrak{X}$ for which $\langle\mathbb{N}, A\rangle_{A \in \mathfrak{X}}$ has a conservative elementary extension.

Theorem 5.2. Assuming $\mathrm{PFA}$, if $\mathfrak{X}$ is a proper Scott set of size $\omega_{1}$, then $\langle\mathbb{N}, A\rangle_{A \in \mathfrak{X}}$ has a conservative elementary extension. 
Proof. Let $\mathcal{L}_{\mathfrak{X}}$ be the language of arithmetic $\mathcal{L}_{A}$ together with unary predicates for sets in $\mathfrak{X}$. Let $G$ be an ultrafilter on $\mathfrak{X}$. We define $\Pi_{\mathfrak{X}} \mathbb{N} / G$, the ultrapower of $\mathbb{N}$ by $G$, to consist of equivalence classes of functions coded in $\mathfrak{X}$. We have to make this modification to the construction of the proof of Theorem 3.4 since the idea of functions coded in the model clearly does not make sense for $\mathbb{N}$. The usual arguments show that we can impose an $\mathcal{L}_{\mathfrak{X}}$ structure on $\Pi_{\mathfrak{X}} \mathbb{N} / G$ and Loś Lemma holds. I will show, by choosing $G$ carefully, that $\left\langle\Pi_{\mathfrak{X}} \mathbb{N} / G, A^{\prime}\right\rangle_{A \in \mathfrak{X}}$ is a conservative extension of $\langle\mathbb{N}, A\rangle_{A \in \mathfrak{X}}$ where $A^{\prime}=\left\{[f]_{G} \in \Pi_{\mathfrak{X}} \mathbb{N} / G \mid\{n \in \mathbb{N} \mid f(n) \in A\} \in G\right\}$. Fix a set $E$ definable in $\left\langle\Pi_{\mathfrak{X}} \mathbb{N} / G, A^{\prime}\right\rangle_{A \in \mathfrak{X}}$ by a formula $\varphi\left(x,[f]_{G}\right)$. Observe that $n \in$ $E \leftrightarrow \Pi_{\mathfrak{X}} \mathbb{N} / G=\varphi\left(n,[f]_{G}\right) \leftrightarrow B_{n}^{\varphi, f}=\{m \in \mathbb{N} \mid \mathbb{N} \models \varphi(n, f(m))\} \in G$. Let $\mathscr{D}_{\varphi, f}=$ $\left\{C \in \mathfrak{X} /\right.$ Fin $\mid C$ decides $\left.\left\langle B_{n}^{\varphi, f} \mid n \in \mathbb{N}\right\rangle\right\}$. The sets $\mathscr{D}_{\varphi, f}$ are dense since $\mathfrak{X}$ is decisive. Clearly if $G$ meets all the $\mathscr{D}_{\varphi, f}$, the ultrapower $\left\langle\Pi_{\mathfrak{X}} \mathbb{N} / G, A^{\prime}\right\rangle_{A \in \mathfrak{X}}$ will be a conservative extension of $\langle\mathbb{N}, A\rangle_{A \in \mathfrak{X}}$. Finally, since $\mathfrak{X}$ has size $\omega_{1}$, there are at most $\omega_{1}$ many formulas $\varphi$ of $\mathcal{L}_{\mathfrak{X}}$ and functions $f$ coded in $\mathfrak{X}$, and hence at most $\omega_{1}$ many dense sets $\mathscr{D}_{\varphi, f}$. So we can find the desired $G$ by PFA

Another open question in the field of models of PA, for which $\mathfrak{X} /$ Fin is relevant, involves the existence of minimal cofinal extensions for uncountable models.

Definition 5.3. Let $M$ be a model of PA, then $M \prec N$ is a minimal extension if it is a proper extension and whenever $M \prec K \prec N$, either $K=M$ or $K=N$.

Theorem 5.4. Every nonstandard countable model of PA has a minimal cofinal extension. (See [?], p. 28)

Gaifman showed that every model of PA, regardless of cardinality, has a minimal end extension [?].

Definition 5.5. Let $\mathfrak{X} \subseteq \mathcal{P}(\mathbb{N})$ be a Boolean algebra. If $U$ is an ultrafilter on $\mathfrak{X}$, we say that $U$ is Ramsey if for every function $f: \mathbb{N} \rightarrow \mathbb{N}$ coded in $\mathfrak{X}$, there is a set $A \in U$ such that $f$ is either $1-1$ or constant on $A$.

Lemma 5.6. If $M$ is a nonstandard model of $\mathrm{PA}$ such that $\mathrm{SSy}(M)$ has a Ramsey ultrafilter, then $M$ has a minimal cofinal extension 8

Proof. Let $U$ be a Ramsey ultrafilter on $\operatorname{SSy}(M)$. The strategy will be to show that the ultrapower $\Pi_{\mathrm{SSy}(M)} M / U$ is a minimal cofinal extension of $M$. The meaning of $\Pi_{\mathrm{SSy}(M)} M / U$ here is identical to the one in the proof of Theorem 3.4. First, observe that for any ultrafilter $\mathrm{U}$, we have $\Pi_{\mathfrak{X}} M / U=\operatorname{Scl}\left(M \cup\left\{[i d]_{U}\right\}\right)$, the Skolem closure of the equivalence class of the identity function together with elements of $M$. This holds since any $[f]_{U}=t\left([i d]_{U}\right)$ where $t$ is the Skolem term defined by $f$ in $M$. Next, observe that such ultrapowers are always cofinal. To see this, fix $[f]_{U} \in \Pi_{\mathfrak{X}} M / U$ and let $a>f(n)$ for all $n \in \mathbb{N}$. Such $a$ exists since $f$ is coded in $M$. Clearly $[f]_{U}<\left[c_{a}\right]_{U}$ where $c_{a}(n)=a$ for all $n \in \mathbb{N}$. These observations hold for any Scott set $\mathfrak{X} \supseteq \operatorname{SSy}(M)$ and, in particular, for $\mathfrak{X}=\operatorname{SSy}(M)$. To show that the extension $\Pi_{\mathrm{SSy}(M)} M / U$ is minimal, we fix $M \prec K \prec \Pi_{\mathfrak{X}} M / U$ and show that $K=M$ or $K=\Pi_{\mathfrak{X}} M / U$. It suffices to see that $[i d]_{U} \in S c l\left(M \cup\left\{[f]_{U}\right\}\right)$ for every $[f]_{U} \in\left(\Pi_{\operatorname{SSy}(M)} M / U\right)-M$. Fix $f: \mathbb{N} \rightarrow M$ and define $g: \mathbb{N} \rightarrow \mathbb{N}$ such that $g(0)=0$ and $g(n)=n$ if $f(n)$ is not equal to $f(m)$ for any $m<n$, or $g(n)=m$ where $m$ is least such that $f(m)=f(n)$. Observe that $g \in \operatorname{SSy}(M)$. Also for any

\footnotetext{
${ }^{7}$ The anonymous referee pointed out that similar arguments have appeared in [?].

${ }^{8}$ This was first proved by [?].
} 
$A \subseteq \mathbb{N}$, the function $g$ is 1-1 or constant on $A$ if and only if $f$ is. Since $U$ is Ramsey, $g$ is either constant or 1-1 on some set $A \in U$. Hence $f$ is either constant or 1-1 on $A$ as well. If $f$ is constant on $A$, then $[f]_{U} \in M$. If $f$ is $1-1$ on $A$, let $s$ be the Skolem term that is the inverse of $f$ on $A$. Then clearly $s\left([f]_{U}\right)=[i d]_{U}$. This completes the argument that $\Pi_{\mathrm{SSy}(M)} M / U$ is a minimal cofinal extension of $M$.

The converse to the above theorem does not hold. If $M$ has a minimal cofinal extension, it does not follow that there is a Ramsey ultrafilter on $\operatorname{SSy}(M)$.9

Theorem 5.7. Assuming PFA, Ramsey ultrafilters exist for proper Scott sets of size $\omega_{1}$. Thus, if $M$ is a model of $\mathrm{PA}$ and $\mathrm{SSy}(M)$ is proper of size $\omega_{1}$, then $M$ has a minimal cofinal extension.

Proof. The existence of a Ramsey ultrafilter involves being able to meet a family of dense sets. To see this, fix $f: \mathbb{N} \rightarrow \mathbb{N}$ and observe that $\mathscr{D}_{f}=\{A \in \operatorname{SSy}(M) /$ Fin $\mid$ $f$ is $1-1$ on $A$ or $f$ is constant on $A\}$ is dense. To see that $\mathscr{D}_{f}$ is dense, actually does not require that $\mathrm{SSy}(M)$ is arithmetically closed.

The proof of Theorem 5.7 shows that any $M$ with a countable standard system will have a minimal cofinal extension since we do not need PFA to construct an ultrafilter meeting countably many dense sets.

\section{WeAKEning the Hypothesis}

There are several ways in which the hypothesis of the main theorem can be modified. PFA is a very strong set theoretic axiom, and therefore it is important to see whether this assumption can be weakened to something that is lower in consistency strength. In fact, there are weaker versions of PFA that still work with the main theorem. It is also possible to make slightly different assumptions on $\mathfrak{X}$. Instead of assuming that $\mathfrak{X}$ is proper, it is sufficient to assume that $\mathfrak{X}$ is the union of a chain of proper Scott sets.

The definition of properness refers to countable structures $M \prec H_{\lambda}$ and the existence of $M$-generic elements for them. If we fix a cardinal $\kappa$ and modify the definition to consider $M$ of size $\kappa$ instead, we will get the notion of $\kappa$-properness. In this extended definition, the notion of properness we considered up to this point becomes $\aleph_{0}$-properness. For example, the $\kappa$-c.c. and $<\kappa$-closed posets are $\kappa$-proper. Hamkins and Johnstone [?] recently proposed a new axiom PFA(c-proper) which states that for every poset $\mathbb{P}$ that is proper and $2^{\omega}$-proper and every collection $\mathcal{D}$ of $\omega_{1}$ many dense subsets of $\mathbb{P}$, there is a filter on $\mathbb{P}$ that meets all of them. $\mathrm{PFA}(\mathfrak{c}$-proper) is much weaker in consistency strength than PFA. While the best large cardinal upper bound on the consistency strength of PFA is a supercompact cardinal, an upper bound for PFA(c-proper) is an unfoldable cardinal [?]. Unfoldable cardinals were defined by Villaveces [?] and are much weaker than measurable cardinals. In fact, unfoldable cardinals are consistent with $V=L$. The axiom $\operatorname{PFA}(\mathfrak{c}$-proper $)$ also decides the size of the continuum is $\omega_{2}[?]$. It is enough for the main theorem to assume that PFA(c-proper) holds:

Theorem 6.1. Assuming PFA(c-proper), every proper Scott set is the standard system of a model of $\mathrm{PA}$.

\footnotetext{
${ }^{9}$ I am grateful to Haim Gaifman for pointing this out, see [?] for a detailed argument.
} 
Proof. Every $\kappa^{+}$-c.c. poset is $\kappa$-proper. It is clear that every Scott set $\mathfrak{X}$ is $\left(2^{\omega}\right)^{+}$c.c.. It follows that every Scott set is $2^{\omega}$-proper. Thus, PFA(c-proper) applies to proper Scott sets.

It is also easy to see that we do not need the whole Scott set $\mathfrak{X}$ to be proper. For the construction, it would suffice if $\mathfrak{X}$ was a union of a chain of proper Scott sets. Call a Scott set piecewise proper if it is the union of a chain of proper Scott sets of size $\leq \omega_{1}$. Under this definition, any arithmetically closed Scott set of size $\leq \omega_{1}$ is trivially piecewise proper since it is the union of a chain of arithmetically closed countable Scott sets. Also, it is clear that a piecewise proper Scott set is arithmetically closed. The modified construction using piecewise proper Scott sets does not require all of PFA but only a much weaker version known as $\mathrm{PFA}^{-}$. The axiom $\mathrm{PFA}^{-}$is the assertion that for every proper poset $\mathbb{P}$ of size $\omega_{1}$ and every collection $\mathcal{D}$ of $\omega_{1}$ many dense subsets of $\mathbb{P}$, there is a filter on $\mathbb{P}$ that meets all of them. $\mathrm{PFA}^{-}$has no large cardinal strength. The axiom is equiconsistent with ZFC [?] (p. 122). This leads to the following modified version of the main theorem:

Theorem 6.2. Assuming $\mathrm{PFA}^{-}$, every piecewise proper Scott set of size $\leq \omega_{2}$ is the standard system of a model of PA.

Proof. It suffices to show that the $\omega_{2}$-Ehrenfeucht Principle holds for piecewise proper Scott sets of size $\omega_{2}$. So suppose $M$ is a model of PA of size $\omega_{1}$ and $\mathfrak{X}$ is a piecewise proper Scott set of size $\omega_{2}$ such that $\operatorname{SSy}(M) \subseteq \mathfrak{X}$. Since $\mathfrak{X}$ is piecewise proper, it is the union of a chain of proper Scott sets $\mathfrak{X}_{\xi}$ for $\xi<\omega_{2}$. Fix any $A \in \mathfrak{X}$, then there is an ordinal $\alpha<\omega_{2}$ such that $\operatorname{SSy}(M)$ and $A$ are contained in $\mathfrak{X}_{\alpha}$. Since $\mathfrak{X}_{\alpha}$ is proper, the $\omega_{2}$-Ehrenfeucht Principle holds for $\mathfrak{X}_{\alpha}$ by Theorem 3.4 Thus, there is $M \prec N$ such that $A \in \operatorname{SSy}(N) \subseteq \mathfrak{X}_{\alpha} \subseteq \mathfrak{X}$.

\section{When is $\mathfrak{X} /$ Fin Proper or Piecewise Proper?}

Here, I give an overview of what is known about the existence of proper and piecewise proper Scott sets. Recall that for a property of posets $\mathscr{P}$, if $\mathfrak{X}$ is arithmetically closed and $\mathfrak{X} /$ Fin has $\mathscr{P}$, I say that $\mathfrak{X}$ has property $\mathscr{P}$.

Theorem 7.1. Any arithmetically closed countable Scott set is proper and $\mathcal{P}(\mathbb{N})$ is proper.

Proof. The class of proper posets includes c.c.c. and countably closed posets. An arithmetically closed countable Scott set is c.c.c. and $\mathcal{P}(\mathbb{N})$ is countably closed.

We are already in a better position than with c.c.c. Scott sets since we have an instance of an uncountable proper Scott set, namely $\mathcal{P}(\mathbb{N})$. This does not, however, give us a new instance of Scott's Problem since we already know by the Compactness Theorem that there are models of PA with standard system $\mathcal{P}(\mathbb{N})$.

The easiest way to show that a poset is proper is to show that it is c.c.c. or countably closed. We already know that if a Scott set is c.c.c., then it is countable (Theorem 2.2). So this condition gives us no new proper Scott sets. It turns out that neither does the countably closed condition.

Theorem 7.2. If $\mathfrak{X}$ is any Scott set such that $\mathfrak{X} /$ Fin is countably closed, then $\mathfrak{X}=\mathcal{P}(\mathbb{N})$. 
Proof. First, I claim that if $\mathfrak{X} /$ Fin is countably closed, then $\mathfrak{X}$ is arithmetically closed. I will show that for every sequence $\left\langle B_{n} \mid n \in \mathbb{N}\right\rangle$ coded in $\mathfrak{X}$, there is $C \in \mathfrak{X}$ deciding $\left\langle B_{n} \mid n \in \mathbb{N}\right\rangle$. This suffices by Theorem 3.3. Fix $\left\langle B_{n} \mid n \in \mathbb{N}\right\rangle$ coded in $\mathfrak{X}$. Define a descending sequence $B_{0}^{*} \geq B_{1}^{*} \geq \cdots \geq B_{n}^{*} \geq \cdots$ of elements of $\mathfrak{X} /$ Fin by induction on $n$ such that $B_{0}^{*}=B_{0}$ and $B_{n+1}^{*}$ is $B_{n}^{*} \cap B_{n+1}$ if this intersection is infinite or $B_{n}^{*} \cap\left(\mathbb{N}-B_{n+1}\right)$ otherwise. By countable closure, there is $C \in \mathfrak{X} /$ Fin below this sequence. Clearly $C$ decides $\left\langle B_{n} \mid n \in \mathbb{N}\right\rangle$. Therefore $\mathfrak{X}$ is arithmetically closed. Now I will show that every $A \subseteq \mathbb{N}$ is in $\mathfrak{X}$. Define $B_{n}=\left\{m \in \mathbb{N} \mid(m)_{n}=\right.$ $\left.\chi_{A}(n)\right\}$ as before. Let $A_{m}=\cap_{n \leq m} B_{n}$ and observe that $A_{0} \geq A_{1} \geq \cdots \geq A_{m} \geq \ldots$ in $\mathfrak{X} /$ Fin. By countable closure, there exists $C \in \mathfrak{X} /$ Fin such that $C \subseteq_{\text {Fin }} A_{m}$ for all $m \in \mathbb{N}$. Thus, $C \subseteq_{\text {Fin }} B_{n}$ for all $n \in \mathbb{N}$. It follows that $A=\{n \in \mathbb{N} \mid$ $\exists m \forall k \in C$ if $k>m$, then $\left.(k)_{n}=1\right\}$. Thus, $A$ is arithmetic in $C$, and hence $A \in \mathfrak{X}$ by arithmetic closure. Since $A$ was arbitrary, this concludes the proof that $\mathfrak{X}=\mathcal{P}(\mathbb{N})$.

The countable closure condition can be weakened slightly. If a poset is just strategically $\omega$-closed, it is enough to imply properness.

Definition 7.3. Let $\mathbb{P}$ be a poset, then $\mathscr{G}_{\mathbb{P}}$ is the following infinite game between players I and II: Player I plays an element $p_{0} \in \mathbb{P}$, and then player II plays $p_{1} \in \mathbb{P}$ such that $p_{0} \geq p_{1}$. Then player I plays $p_{1} \geq p_{2}$ and player II plays $p_{2} \geq p_{3}$. Player I and II alternate in this fashion for $\omega$ steps to end up with the descending sequence $p_{0} \geq p_{1} \geq p_{2} \geq \ldots \geq p_{n} \geq \ldots$. Player II wins if the sequence has a lower bound in $\mathbb{P}$. Otherwise, player I wins. A poset $\mathbb{P}$ is strategically $\omega$-closed if player II has a winning strategy in the game $\mathscr{G}_{\mathbb{P}}$.

Observe that if $\mathfrak{X}$ is a Scott set such that $\mathfrak{X} /$ Fin is strategically $\omega$-closed, then $\mathfrak{X}$ has to be arithmetically closed. To see this, suppose that $\mathfrak{X} /$ Fin is strategically $\omega$-closed and $\left\langle B_{n} \mid n \in \mathbb{N}\right\rangle$ is a sequence coded in $\mathfrak{X}$. We will find $C \in \mathfrak{X} /$ Fin deciding the sequence by having player I play either $B_{n}$ or $\mathbb{N}-B_{n}$ intersected with the previous move of player II at the $n^{\text {th }}$ step of the game. It is not known whether there are Scott sets that are strategically $\omega$-closed but not countably closed.

One might wonder at this point whether it is possibly the case that a Scott set is proper only when it is countable or $\mathcal{P}(\mathbb{N})$ and a Scott set is piecewise proper only when it is of size $\leq \omega_{1}$. In a forthcoming paper [?], I show the following results about the existence of proper and piecewise proper Scott sets.

First, I show that one can obtain uncountable proper Scott sets other that $\mathcal{P}(\mathbb{N})$ by considering when the $\mathcal{P}(\mathbb{N})$ of $V$ remains proper in a generic extension after forcing to add new reals.

Theorem 7.4. If $\mathrm{CH}$ holds and $\mathbb{P}$ is a c.c.c. poset, then $\mathcal{P}^{V}(\mathbb{N}) /$ Fin remains proper in $V[g]$ where $g \subseteq \mathbb{P}$ is $V$-generic.

In particular, if $\mathrm{CH}$ holds in $V$ and we force to add a Cohen real, then the $\mathcal{P}(\mathbb{N})$ of $V$ will be a proper Scott set in the generic extension.

It is also possible to force the existence of many proper Scott sets of size $\omega_{1}$ and piecewise proper Scott sets of size $\omega_{2}$.

Theorem 7.5. There is a generic extension of $V$ by a c.c.c. poset, which contains continuum many proper Scott sets of size $\omega_{1}$.

Theorem 7.6. There is a generic extension of $V$ by a c.c.c. poset, which contains continuum many piecewise proper Scott sets of size $\omega_{2}$. 
Finally, Enayat showed in [?] that ZFC proves the existence of an arithmetically closed Scott set of size $\omega_{1}$ which is not proper.

Theorem 7.7 (Enayat, 2006). There is an arithmetically closed Scott set $\mathfrak{X}$ such that $\mathfrak{X} /$ Fin collapses $\omega_{1}$. Hence $\mathfrak{X}$ is not proper.

Clearly $\mathfrak{X} /$ Fin cannot be proper since proper posets preserve $\omega_{1}$.

Recall that any arithmetically closed Scott set of size $\omega_{1}$ is trivially piecewise proper. It follows that there are piecewise proper Scott sets which are not proper. It is not clear whether every proper Scott has to be piecewise proper.

\section{Questions}

Question 8.1. Can ZFC or ZFC + PFA prove the existence of an uncountable proper Scott set other than $\mathcal{P}(\mathbb{N})$ ?

Question 8.2. Is it consistent with ZFC that there are proper Scott sets of size $\omega_{2}$ other than $\mathcal{P}(\mathbb{N})$ ?

Question 8.3. Are there Scott sets that are strategically $\omega$-closed but not countably closed?

Question 8.4. Does the $\omega_{2}$-Ehrenfeucht Principle hold or fail (consistently)?

Question 8.5. Does the $\omega_{2}$-Ehrenfeucht Principle hold for models with a countable standard system? That is, can we remove the assumption of arithmetic closure from Theorem 4.1?

New York City College of Technology (CUNy), Mathematics, 300 Jay Street, BrookLYN, NY 11201 USA

E-mail address: vgitman@nylogic.org 